\title{
Psicooncología
}

ISSN: $1696-7240$

\section{Cáncer parental: díptico de soporte para padres y madres recién diag- nosticados}

Sònia Fuentes Sanmartín; Milagros Barez Villoria²; Lydia Caba Velaㅎ María Luisa de Cáceres Zurita $^{4}$

\section{Parental cancer: support diptych for newly diagnosed parents and mothers}

Comunicar las malas noticias relacionadas con la aparición de la enfermedad supone para muchos pacientes oncológicos una situación de gran dificultad y sufrimiento. Muchos de ellos son padres de niños y adolescentes y con frecuencia plantean cuestiones a los profesionales que componen el equipo de cuidados oncológicos, sobre cómo hablar a sus hijos de la enfermedad.

Los estudios realizados previamente en el campo de los cuidados paliativos y en la atención al duelo, demuestran que hablar de la muerte abiertamente todavía supone un tabú en la cultura occidental, y más aún cuando se trata de hablar de la muerte de un familiar a los niños ${ }^{(1-5)}$. Estos estudios expresan las dificultades que aparecen en el seno familiar cuando se dan estas situaciones que generan tanto sufrimiento. Los condicionamientos culturales influyen, sin duda, en el momento en que un adulto diagnosticado de cáncer debe informar a sus hijos menores sobre el diagnóstico de la enfermedad, generándose dudas y cuestiones respecto a la conveniencia de informar o sobre la mejor manera de hacerlo ${ }^{(6,7)}$. Hay gran cantidad de estudios centrados en los efectos psicológicos que el cáncer parental puede ocasionar en los niños y adolescentes $^{(8,9)}$, así como en las secuelas que pueden mantenerse a largo plazo si no se les incorpora a estos procesos vividos en el seno de la familia ${ }^{(10,11)}$.

1 Sònia Fuentes Sanmartín. Unidad de Psicooncología Instituto Catalán de Oncología, Badalona. Hospital Universitario GermansTrias i Pujol/ Hospital de Mataró.

E-mail: sfuentes@iconcologia.net

2 Milagros Barez Villoria. Unidad de Psicooncología Instituto Catalán de Oncología, Badalona. Hospital Universitario GermansTrias i Pujol.

E-mail: mbarez@iconcologia.net

3 Lydia Caba Vela Unidad de Psicooncología Instituto Catalán de Oncología, Girona. Hospital St. Jaume de Calella E-mail: 1caba@iconcologia.net

4 Maria Luisa de Cáceres Zurita. Unidad de Psicooncología Instituto Catalán de Oncología, Badalona. Hospital Universitario GermansTrias i Pujol.

E-mail: mldecaceres@iconcologia.net

* Dirección de correspondencia: Sònia Fuentes Sanmartín. Unidad de Psicooncología. Instituto Catalán de Oncología -Badalona. Hospital Universitario Germans Trias i Pujol. Carretera del Canyet, s/n. 08916 - Badalona. E-mail: sfuentes@iconcologia.net 
Teniendo en cuenta las diversas investigaciones anteriormente citadas y pretendiendo seguir la misma línea, hemos elaborado un documento sencillo, práctico y útil, realizado en forma de díptico bilingüe (catalán, castellano), con formato DIN A4, para poder hacerlo llegar a los pacientes oncológicos y a sus familiares. Este documento fue planteado a raíz de una necesidad detectada desde hace años en el trabajo de nuestra Unidad de Psicooncología con el objetivo principal de favorecer la comunicación entre los padres enfermos y sus hijos, con el fin de abordar dudas, angustias y reflexiones muy frecuentemente omitidas en las consultas médicas, donde se suele priorizar la atención a la parte más orgánica de la persona. Sobre esta temática en concreto fuimos implicando a diversos profesionales del equipo, generando formación y sensibilidad sobre el tema a oncólogos, hematólogos, enfermeras, trabajadoras sociales, etc... Asimismo, desde la Unidad de Psicooncología del Instituto Catalán de Oncología (ICO) de Badalona, se han llevado a cabo previamente varias investigaciones sobre el proceso de comunicación entre padres con cáncer y sus hijos ${ }^{(12,13)}$ En el territorio español se encuentran también otros equipos con la misma motivación por este tema ${ }^{(1,14)}$.

Como ya hemos comentado anteriormente, el objetivo principal del díptico es favorecer la comunicación sobre la enfermedad entre los padres recién diagnosticados de cáncer y los hijos menores a su cargo. Así, este sencillo documento contiene una serie de recomendaciones básicas que pretenden servir de guía en un momento de gran dificultad como es el diagnóstico de una enfermedad oncológica. Las describimos a continuación:

Edad del niño: Se invita a reflexionar sobre la importancia de la edad del niño, explicando brevemente las diferencias entre los niños más pequeños y los más mayores en cuanto a la conciencia de enfermedad y por lo tanto también en relación a la comprensión sobre la situación que vive su padre/madre enfermo.

Uso de la palabra cáncer: Es frecuente que la palabra "cáncer" asuste y provoque miedos, temores y resistencias a ser utilizada en las conversaciones con los niños. Recomendamos, si ese es el caso, utilizar otra palabra sinónima que ayude a iniciar el proceso de comunicación entre el padre enfermo y su hijo, con la aclaración de no mentir nunca ante las preguntas que generen los menores.

Dosificación de la información: Igual que sabemos que es necesario incorporar a los niños en los procesos de enfermedad de sus padres, también sabemos que no es necesario dar toda la información que posee el adulto a un niño, y recomendamos hacerlo de forma secuenciada, dosificando especialmente, las malas noticias.

Expresión emocional: Recomendamos dignificar la expresión emocional de los pacientes ante sus hijos. Muy a menudo, los padres temen emocionarse y llorar ante sus niños.

Efectos secundarios de los tratamientos y alteración de las rutinas familiares: Es necesario informar previamente a la aparición de los principales efectos secundarios de los tratamientos oncológicos, especialmente los más llamativos (alopecia, astenia, pérdida de energía, etc...)

Comunicación con la escuela: Es de crucial importancia informar al tutor del niño sobre la situación que se está viviendo en la familia. La escuela siempre ofrece recursos a los niños que necesitan ayuda. 


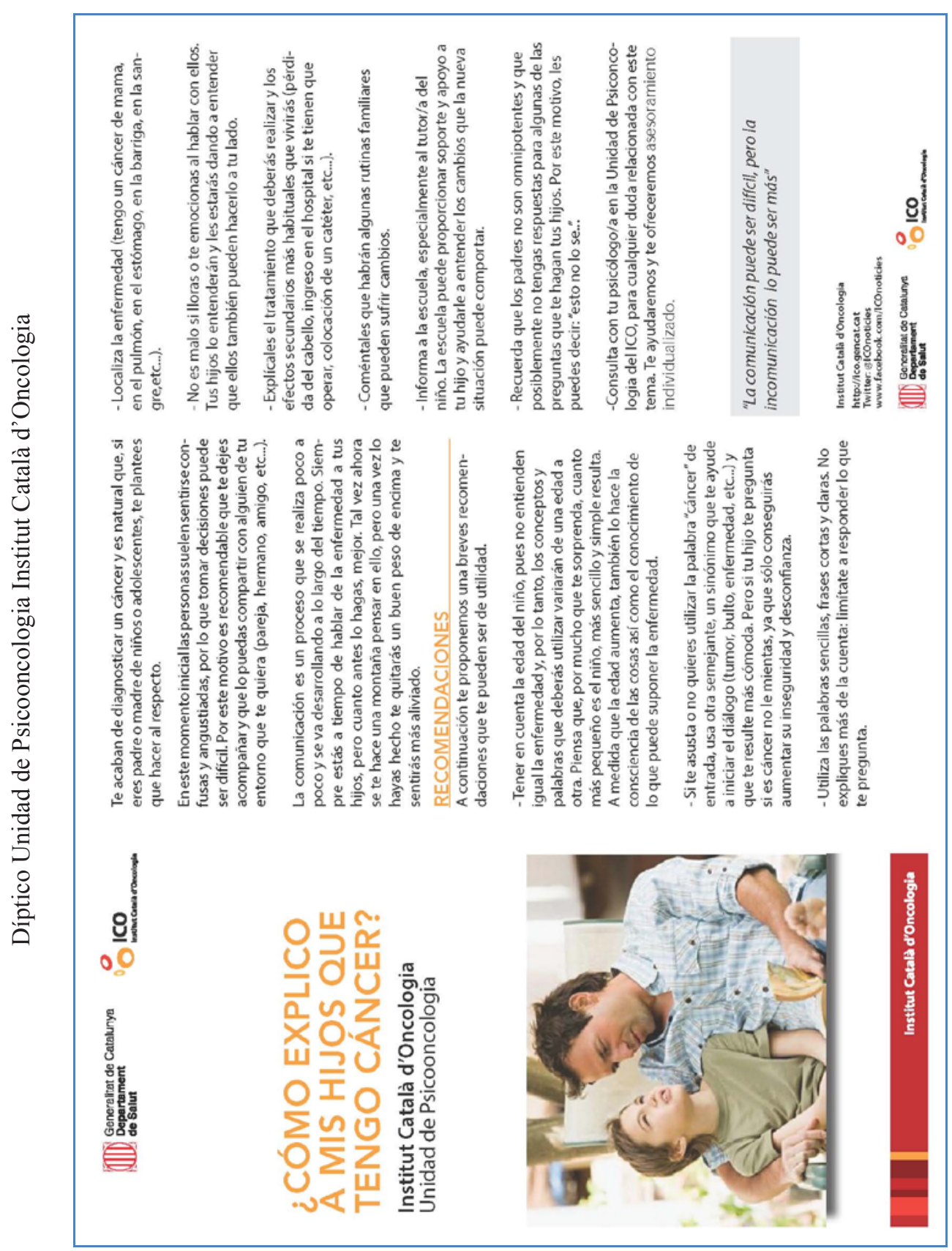


Este documento se distribuyó en las salas de espera de los diferentes servicios oncológicos de nuestro centro: Servicio de Oncología Médica, Servicio de Oncología Radioterápica y planta de Hospitalización de Oncología, Hemato-Oncologia y Cuidados Paliativos. También se distribuyeron ejemplares en las consultas externas de Oncología, Hematología y en la consulta de enfermería del Hospital de Día, como material de soporte para los profesionales que atienden a los pacientes en estos espacios (médicos y enfermeras). Para facilitar y ampliar el acceso al documento a cuantos más pacientes y familiares, se distribuyó también entre las diferentes Unidades de Psicooncología ( 3 en total), de todos los centros ICO de nuestra área de influencia.

Según nuestra experiencia, las ventajas de la creación y distribución de este documento han mostrado ser, por un lado la gran utilidad que ha representado para los pacientes afectados, así como para sus familiares, y por otro lado hemos detectado que este díptico también supone una buena ayuda para los profesionales que, sin ser psicólogos, afrontan cuestiones relacionadas con la comunicación planteadas por los pacientes en las primeras visitas de contacto, pues con este escrito pueden facilitar una primera orientación al paciente oncológico, así como valorar la posibilidad y necesidad de derivación a nuestra unidad de Psicooncología.

Finalmente, pretendemos reconocer y validar el derecho de los niños a ser tenidos en cuenta y a ser atendidos en cualquier situación de riesgo y vulnerabilidad familiar.

\section{Referencias bibliográficas}

1. Mesquida V, Seijas R, Rodriguez M. Los niños ante la pérdida de uno de los progenitores: revisión de pautas de comunicación eficaces. Psicooncología 2015; 12: 417-29. Doi: 10.5209/rev_PSIC.2015.v12.n2-3.51019

2. Christ, G. Healing children's grief: Surviving a parent's death from cancer. New York: Oxford University Press, 2000.

3. Christ G, Christ A. Current appraches to helping children cope with a parent's terminal illness. CA Cancer J Clin 2006; 56: 197-212. Doi: 10.3322/canjclin.56.4.197

4. Fearnley, R. Communicating with children. When a parent is at the end of life.London: Jessica Kingsley Publishers, 2012.

5. Muriel A, Rauch P. Talking with families and children about the death of a parent. En Hanks G, Cherny N, Christakis N, Fallon M, Stein Kaasa R, editors. Oxford Textbook of Palliative Medicine. New York: Oxford University Press, 2010.p. 342-347.

6. Fuentes $\mathrm{S}$. Comunicació en càncer: Anàlisi de les preocupacions i dels recursos dels pares recent diagnosticats al comunicar la malaltia als seus fills. (Tesis doctoral). Barcelona: Universidad Autónoma de Barcelona, 2015.

7. Rodríguez AM. Els nens y adolescents davant el càncer avançat o terminal dels pares. (Tesi Doctoral). Barcelona: Universitat Autònoma de Barcelona, 2013.

8. Phillips F. Adolescents living with a parent with advanced cancer: a review of the literature. Psychooncology 2014;12: 1323-9. doi: 10.1002/pon.3570

9. Huizinga G, Visser A, van der Graaf W, Hoekstra H, Stewart R, Hoekstra-Weebers J. Family-oriented multilevel study of the psychological functioning of adolescent children having a mother with cancer. Psychooncology 2011; 20: 730-7. Doi: 10.1002/ pon. 1779 
10. Huizinga G, Visser A, van der Graaf W, Hoekstra H, Hoekstra-Weebers J. The quality of communication between parent and adolescent children in the case of parental cancer. Ann Oncol 2005; 16: 1956-65.

11. Huizinga G, Visser A, Zelders-Steyn Y, Teule J, Reijneveld S, Roodbol P. Psychological impact of having a parent with cancer. Eur J Cancer 2011; 47 (3 Suppl), S239-S246.

12. Fuentes S, Blasco T. Preocupaciones de los pacientes con cáncer de mama que reciben atención psicooncológica. Psicooncología 2010; 7: 51-60.

13. Fuentes S, Blasco T. Factores predictores de la adaptación a la enfermedad en pacientes recién diagnosticadas de cáncer de mama que acuden a un servicio de psicooncología. An Psicol 2012; 28: 736-42.

14. Sordo T, Rubio I, Peralta M, Ángeles M, i Ospina L. ¿Cómo se lo digo a mi hijo/a? Psicooncologia 2014; 11(supl. 1):1-83. 